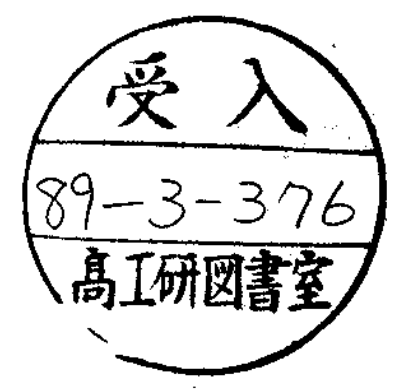

CERN-TII.5305/89

\title{
A PARTICLE MECHANICS DESCRIPTION OF ANTISYMMETRIC TENSOR FIELDS
}

\author{
Paul llowe $\dagger$ \\ CERN, Geneva, Switzerland \\ Silvia Penati \\ Physics Department, Brandeis University; Waltham MA 02254 \\ Mario Pernici and Pauł K. Townsend \\ CERN, Crencta, Switzerland
}

\begin{abstract}
$\triangle H S I R A C I$
In a previous work we discussed the action for a massless relativistic point particle with a gauged $\mathrm{N}$-extended worldline supersymmetry that yiclds, upon quantization, a relativistic wave equation for pure spin $N / 2$. Here we present further details, emphasizing the $N=2$ particle model for which a wave funclion with ()$(2)$ charge $q$ can be interpreted as the ficld strength of a (q-1)-form gauge potential. We present extensions of this model that yield field equations for massless and massice antisymmetric tensors in arbitrary space-time dimension $d$. We show how to obtain chirality and generalized self-duality conditions.
\end{abstract}

$\dagger$ Permanent address : Department of Mathematics, King's College, London

$\ddagger$ Permanent address : DAMTP, Univ. of Cambridge, England

CER.N-TH.5305/89

February 1989. 


\section{Introduction}

Relativistic free ficld equations for massless particles of arbitrary spin can be obtained from the quantization of point particle models. The Klein-Gordon equation comes from the spinless particle, the Klein-Gordon operator $p^{2}$ being the generalor of reparametrizations of the particle worldine. The I)irac equation is similarly obtained from the spinning particle action which has $N=1$ worldline supersymmetry [1,2]. 'The anticommuting classical variables $\lambda^{\prime \prime}$ become the Dirac matrices on quantization and the generator of supersymmetry transformations becomes the Dirac operator. This model can be extended to describe particles of spin greater than $\frac{1}{2}[3,4,5]$. The extended model has local $\mathrm{N}$ extended worldline supersymmetry and a local $(N)$ gauge invariance. For a particle moving in Minkowski space-time with coordinates $x^{\mu}$ and momentum $p_{\mu}$ the first-order form of the action is

$$
S=\int_{0}^{1} d t\left\{\dot{x}^{\mu} p_{\mu}+\frac{i}{2} \lambda_{i}^{\mu} \dot{\lambda}_{i}^{\nu} \eta_{\mu^{\prime \nu}}-\frac{1}{2} e \eta^{\mu \nu} p_{\mu} p_{\nu}-i \psi_{i} \lambda_{i}^{\prime \prime} p_{\mu}-\frac{i}{2} \int_{i j} \lambda_{i}^{\mu} \lambda_{j}^{\nu} \eta_{\mu \nu}\right\}
$$

where the $\lambda_{i}^{\mu}(t) i=1, \ldots N$ are the supersymmetry partners of $x^{\mu}(t), \quad e(t)$ and $\psi_{i}(t)$ are "worldline supergravity ficlds" and $f_{i j}(l)$ is the $S()(N)$ gange "ficld". 'The gange ficlds $e, \psi_{i}$, and $f_{i j}$ are the Iagrange multipliers for the comstraints which, when imposed on the particle's quantum wave function, yicld a relativistic wase equation for a pure spin $N / 2$ particle (in four elimensions). Because the action also has a d-elimensional conformal invariance these wave equalions are in fact the conformal wave cquations for arbitrary spin. This has been noted independently by Siegel [6], who has further shown that all conformal wave equations are oblained in this way.

In this paper we shall emphasize the $N=2$ molel. In this case we can add to the action (1.1) a "Chern-Simons" term proportional to

$$
\int_{0}^{1} d l \varepsilon^{i j} f_{i j}
$$


which is not possible for $N>2$ because the internal symmetry group is then non- $\Lambda$ belian. Without the Chern-Simons term, the $N=2$ wave-function is necessarily a $\frac{d}{2}$-form, which is further constrained to be harmonic. This means that for $\mathrm{d}$ odd the wave-function must vanish. As we shall show, this can be understood in the context of path-integral quantization as a consequence of a global $S O(2)$ anomaly. With the inclusion of (1.2) with coefficient $\left(q-\frac{d}{2}\right)$ the wave-function becomes a harmonic $q$-form. Since $q$ is an integer this means that for $d$ odd a non-vanishing wave-function requires a non-vanishing ChernSimons coefficient. Since the Chern-Simons term breaks $O(2)$ to $S O(2)$ and conformal invariance to dilatation invariance, the global anomaly for d odd can be viewed as a clash between the rigid $O(2)$ and conformal symmetrics and the local $\mathrm{SO}(2)$ invariance.

Another property of the $N=2$ model is that it can be formulated in superspace (whereas the required auxiliary ficlds are not known for $N>2$ ). Particle models for massive antisymmetric tensor fields in d dimensions can be obtained by a particle mechanics analogue of dimensional reduction à la Scherk and Schwarz [7] from the $N=2$ massless model in $(d+1)$ dimensions. If space-time has a compact isometry group $G$ the point particle action will have a rigid $G$-invariance. If this symmetry is gauged, the particle's wave function will have to salisfy the additional constraint that it be a $G$ singlet. The simplest case is when $G$ is the $U(1)$ isometry group associalcd with translation around an $S^{1}$ factor of a $(d+1)$-dimensional spacetime. Then the requirement that the wave function be a $U(1)$ singlet eftects an (ordinary) dimensional reduction, producing a set of d-dimensional massless wave cquations. Because $U(1)$ is $\Lambda$ belian a modification of the particle action is possible for which the wave-function acquires a non-zero $U(1)$ charge. The resulting d-dimensional equalions are those of a massive antisymmetric tensor.

Siegel and Zwiebach have mentioned how a chirality constraint on a Dirac spinor can be incorporated into a particle model [8] by including extra generalors that enlarge the $N=1$ supersymmetry algebra ${ }^{*}$. At the quantum level the new algebra becomes that of an

\footnotetext{
* A slightly different approach was described in [9]
} 
$N=2$ supersymmetry (but the wavefunction remains a spinor, rather than the bispinor of the $N=2$ models that we have been discussing above). We show how this can be extended to the $N=2$ models for antisymmetric lensors in $d=2 \bmod 4$ to incorporate a self-duality constraint on a $\frac{d}{2}$ form wave funclion. The algebra has six Grassman odd generators in general and is easily investigated in the language of differential forms. For $d=2$ the algebra reduces to an $N=4$ supersymmetry algebra with an additional gauged $O(1,1)$ invariance.

\section{Particle actions for arbitrary spin}

Quantization of the particle with action (1.1) leads to the (anti)commutation relations

$$
\left[x^{\mu}, p_{\nu}\right]=i \delta_{\nu}^{\mu} \quad\left\{\lambda_{i}^{\mu}, \lambda_{j}^{\nu}\right\}=\eta^{\mu \nu} \delta_{i j}
$$

The $\lambda$-relations can be realized by matrices in the space $\otimes^{N} I_{1}$ where, supposing $d$ to be

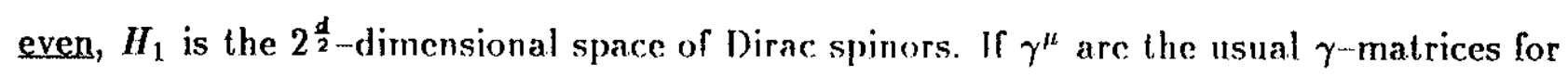
$H_{1}$ and

$$
\gamma_{\star}=i^{\frac{d-2}{2}} \gamma^{0} \gamma^{1} \ldots \gamma^{d-1}
$$

which satisfies $\gamma_{\star}^{2}=1$ and $\left\{\gamma_{\star}, \gamma^{\mu}\right\}=0$, we can represent the operators $\lambda_{i}^{\prime \prime}$ by

$$
\lambda_{i}^{\mu}=\frac{1}{\sqrt{2}} \gamma_{\star} \otimes \ldots \otimes \gamma_{\star} \otimes \mathbf{I}_{(i)}^{\prime \prime} \otimes \mathbb{I} \otimes \ldots \otimes \mathbf{L}
$$

where $\Gamma_{(i)}^{\mu}$ can be chosen, for each value of $i=1, \ldots, N$, to be cillice $\gamma^{\mu}$ or $i \gamma_{\star} \gamma^{\mu}$.

With this realization of the anticommutation relations the wave function is a multispinor $\Psi_{\alpha_{1} \ldots \alpha_{N}}$. The $O(N)$ gauge ficld $f_{i j}$ imposes the algebraic constraint

$$
\left(\gamma_{\mu} \mathrm{l} \gamma^{\mu}\right)^{\alpha_{i} \alpha_{j}} \Psi_{\alpha_{1} \ldots \alpha_{i} \ldots \alpha_{j} \ldots \alpha_{N}}=0
$$

where $\Gamma$ is any Dirac matrix (with entries $\Gamma_{\alpha}^{\beta}$ ). We are using here the notation that $\Gamma^{\alpha \beta}=C^{\alpha \gamma} \Gamma_{\gamma}^{\beta}$ with $C$ the charge conjugation matrix. Let us introduce the further notation 
$\Gamma^{(n)}$ for any of the $\Gamma$-matrices $\gamma^{\mu_{1} \ldots \mu_{n}}$; then $\left(\Gamma^{(n)}\right)^{\alpha \beta}$ is symmetric or antisymmetric in spinor indices, depending on $N$ and $d$. From the identily

$$
\gamma_{\mu} \Gamma^{(n)} \gamma^{\mu}=(-1)^{n}(d-2 n) \Gamma^{(n)}
$$

we see that (2.4) yields the constraints

$$
\left(\mathrm{I}^{(n)}\right)^{\alpha_{i} \alpha_{j}} \Psi_{\alpha_{1} \ldots \alpha_{i} \ldots \alpha_{j} \ldots \alpha_{N}}=0 \quad n \neq \frac{d}{2}
$$

For $d$ odd the representation (2.3) fails because $\gamma_{\star}$ will commute with $\gamma^{\mu}$. By taking $\gamma^{\mu}$ to be a reducible matrix representation of the Clilford algebra generators we could find a suitable replacement for $\gamma_{\star}$ but the constraint (2.6) would still hold and this implies that $\Psi=0$ for $d$ odd. We shall sec shortly that for $N=2$ these difficulties can be overcome, and that allowing for an SO(2) Chern-Simons term cnalbles us to obtain the wave equation for antisymmetric tensors of arbitrary rank in any spacelime dimension.

The supergravity ficlds $e$ and $\psi_{i}$ impose the dynamical constraints

$$
\begin{aligned}
& p^{2} \Psi_{\alpha_{1} \ldots \gamma_{N}}=0 \\
& p_{\beta_{i}}^{\alpha_{i}} \Psi_{\alpha_{1} \ldots \alpha_{i} \ldots \alpha_{N}}=0 \quad \forall i
\end{aligned}
$$

on the wave function. For $d=4$ these are the massless Bargmann-Wigner relativistic wave equations for a pure spin $\frac{N}{2}$ fick strength. In particular, for $N=2$ the wave function is $F_{\mu \nu}$ (the Maxwell ficld strength tensor), and the cquations (2.7) are equivalent to the Bianchi "identity" $\partial_{[\mu} F_{\nu \rho]}=0$ and the ficld cquation $\partial^{\mu} F_{\mu \nu}=0$.

For $N=2 M$ in four dimensions, the wave function can be expressed in the equivalent form $\Psi_{\mu_{1} \nu_{1}, \mu_{2} \nu_{2}, \ldots, \mu_{M} \nu_{M}}^{(2 M, 2)}$ with antisymmetry in cach pair, and total symmetry under interchange of pairs. In adelition it is totally traceless and satisfies

$$
\Psi_{\left[\mu_{1} \nu_{1}, \mu_{2}\right] \nu_{2}, \ldots, \mu_{M} \nu_{M}}=0
$$


The differential constraints are then

$$
\begin{aligned}
& \partial^{\mu} \Psi_{\mu \nu_{1}, \mu_{2} \nu_{2}, \ldots, \mu_{M} \nu_{M}}^{(2 M, 2)}=0 \\
& \partial_{[\rho} \Psi_{\left.\mu_{1} \nu_{1}\right]_{, H_{2} \nu_{2}, \ldots, \mu_{A} \nu_{M}}^{(2 M I, 2)}}^{(2 M}=0
\end{aligned}
$$

For $N=4$, for example, we recover the lineariyed Finstein equations expressed in terms of the Weyl tensor. More generally, in $d=2 n$ dimensions and $N=2 M$, the basic index "block" has n antisymmetrized indices, and the wavefunctions are symmetrized on $M$ blocks, i.e.

$$
\Psi_{\mu_{11} \ldots \mu_{1 n}, \mu_{21} \ldots \mu_{2 n}, \ldots, \mu_{M} \mu_{1} \ldots \mu_{M / n}}^{(2 M, n)}=\Psi_{\left(\left[\mu_{11} \ldots \mu_{1 n}\right],\left[\mu_{21}, \ldots, \nu_{2 n}\right], \ldots,\left[\mu_{M} \ldots \nu_{M N}\right]\right)}^{(2 M I, n)}
$$

where the symmetrization refers to the blocks of $n$ indices. In addition

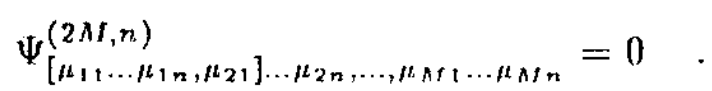

Again, the $\Psi^{(2 M, n)}$ 's are totally traceless and obey equations similar to (2.9). These forms for the wave-functions can be summarized by the $O(n)$ Young lableaux with $n$ rows and $N / 2$ columns, where irreducibility also implies tracelessness. For $N=2 M+1$ in $d=2 n$ dimensions the wavefunction is a tensor-spinor with tensor indices of the same form as $\Psi^{(2 M, n)}$. In this case there is also the $\gamma$-traccless condition

$$
\gamma^{\mu_{11}} \Psi_{\mu_{11} \ldots \mu_{1 n}, \mu_{21} \ldots \mu_{2 n}, \ldots, \mu_{M 11} \ldots \mu_{M / n}}^{(2 N+1, n)}=0
$$

We shall now consider the $N=2$ case in more detail. The action is

$$
\begin{aligned}
S= & \int d t\left\{\dot{x}^{\mu} p_{\mu}+\frac{i}{2} \lambda_{i}^{\mu} \dot{\lambda}_{i}^{\nu} \eta_{\mu \nu}-\frac{1}{2} c \eta^{\prime \nu \nu} p_{\mu} p_{\nu}-i \psi_{i} \lambda_{i}^{\mu} p_{\mu}+\right. \\
& \left.+\int\left(-\frac{i}{2} \varepsilon^{i j} \lambda_{i}^{\mu} \lambda_{j}^{\nu} \eta_{\mu \nu}-\left(q-\frac{d}{2}\right)\right)\right\} .
\end{aligned}
$$

This action differs from the specialization of (1.1) to $N=2$ by the addition of the ( $q-$ $\frac{d}{2}$ ) term. This addition is consistent with supersymmetry because the supersymmetry 
variation of $f$ vanishes. It is also consistent, with worldline diffeomorphism and $S O(2)$ invariance because for these symmetries $\delta f$ is a total derivative. For $N>2$ the $S O(N)$ variation of $f_{i j}$ is not a total derivative, so this modification is no longer possible. We are using units with $\hbar=1$ here; if we were to restore $h$, then the Chern-Simons term $-f(q-d / 2)$ would be multiplied by $h$ and would therefore vanish in the classical limit. Note that, with the exception of the Chern-Simons term, all terms of the action are invariant under $O(2)$, rather than $S O(2)$; the additional transformation is $\lambda_{1} \rightarrow \lambda_{1}, \lambda_{2} \rightarrow-\lambda_{2}, \psi_{1} \rightarrow$ $\psi_{1}, \psi_{2} \rightarrow-\psi_{2}, e \rightarrow e, f \rightarrow-f, x \rightarrow x, p \rightarrow p$

It will prove convenient to introduce the variables

$$
\begin{array}{ll}
\xi^{\mu}=\frac{1}{\sqrt{2}}\left(\lambda_{1}^{\mu}+i \lambda_{2}^{\prime \prime}\right) & \bar{\xi}^{\mu}=\frac{1}{\sqrt{2}}\left(\lambda_{1}^{\mu}-i \lambda_{2}^{\prime \prime}\right) \\
\psi=\frac{1}{\sqrt{2}}\left(\psi_{1}+i \psi_{2}\right) & \bar{\psi}=\frac{1}{\sqrt{2}}\left(\psi_{1}-i \psi_{2}\right)
\end{array}
$$

in terms of which the action reads

$$
\begin{gathered}
S=\int d t\left\{\dot{x}^{\mu} p_{\mu}+i \bar{\xi}^{\mu} \dot{\xi}^{\nu} \eta_{\mu \nu}-i \psi \bar{\xi}^{\mu} p_{\mu}-i \bar{\psi} \xi^{\mu} p_{\mu}-\frac{1}{2} e p^{2}\right. \\
\left.+\int\left(\frac{1}{2}\left[\xi^{\mu}, \dot{\xi}^{\nu}\right] \eta_{\mu \nu}-\left(q-\frac{d}{2}\right)\right)\right\}
\end{gathered}
$$

Upon quantization, the $\xi, \vec{\xi}$ variables satisfy the anticommutation relations

$$
\begin{aligned}
& \left\{\xi^{\prime \prime}, \xi^{\prime \prime}\right\}=\left\{\bar{\xi}^{\mu}, \xi^{\nu}\right\}=0 \\
& \left\{\xi^{\mu}, \ddot{\xi}^{\prime \prime}\right\}=\eta^{\prime \prime \prime}
\end{aligned}
$$

Since the $\bar{\xi}^{\mu}$ are a set of mutually anticommuling opcrators they can be diagonalized on a basis of eigenstates $|\bar{\alpha}\rangle$ for which the cigenvalues $\bar{\alpha}^{\prime \prime}$ of $\xi^{\prime \prime}$ are anticommuting. Thus

$$
\bar{\xi}^{\prime \prime}|\ddot{\alpha}\rangle=\bar{\alpha}^{\prime}|\ddot{\alpha}\rangle
$$

We are using here the notation of [10] to which we refer for further details of properties of the fermion coherent states $|\bar{\alpha}\rangle$. The wave function of the $N=2$ particle is

$$
(\langle x|\otimes\langle\alpha|) \mid \Psi\rangle=\Psi(x, \alpha)
$$


which can be expanded as a power series in $\alpha^{\prime \prime}$. Thus

$$
\begin{aligned}
\Psi= & F(x)+\alpha^{\mu} F_{\mu}(x)+\frac{1}{2} \alpha^{\mu} \alpha^{\nu} F_{\mu \nu}(x)+\ldots \\
& +\frac{1}{p !} \alpha^{\mu_{1}} \ldots \alpha^{\mu_{p}} F_{\mu_{1} \ldots \mu_{p}}(x)+\ldots+\frac{1}{d !} \alpha^{\mu_{1}} \ldots \alpha^{\mu_{d}} F_{\mu_{1} \ldots \mu_{d}}(x)
\end{aligned}
$$

i.e. $\Psi$ can be viewed as an inhomogencous differential form on Minkowski space. The constraint imposed by $f$ can now be written in the form

$$
(\xi \cdot \bar{\xi}-q)|\Psi\rangle=0
$$

which states that. $|\Psi\rangle$ has $O(2)$ charge $q$. Equation $(2.20)$ is equivalent to

$$
\alpha^{\prime \prime} \frac{\partial}{\partial \alpha^{\prime \prime}} \Psi(x, \alpha)=q \Psi(x, \alpha)
$$

which is solved by wriling $\Psi(x, \alpha)$ as

$$
\Psi(x, \alpha)=\frac{1}{q !} \alpha^{\mu_{1}} \ldots \alpha^{\mu_{q}} F_{\mu_{1} \ldots \mu_{q}}(x)
$$

The two independent constraints imposed by $\psi_{i}$ can now be written as

$$
\xi \cdot p|\Psi\rangle=0 \quad \xi \cdot p|\Psi\rangle=0
$$

which are equivalent to

$$
\partial_{\left[\mu_{1}\right.} F_{\left.\mu_{2} \ldots \mu_{q+1}\right]}=0 \quad \partial^{\mu_{1}} F_{\mu_{1} \ldots \mu_{\eta}}=0
$$

respectively. The first equation is solved by $F_{\mu_{1} \ldots \mu_{q}}=q \partial_{\left[\mu_{1}\right.} A_{\left.\mu_{2} \ldots \mu_{q}\right]}$ and the second is then the usual field equation for the $(q-1)$ 'th rank antisymmetric tensor gange potential $A$. Observe that this analysis holds for $d$ cven and $d$ odd.

Because of the anticormmutation relations (2.16) the $\frac{d}{2}$ term in the $S O(2)$ constraint is cancelled when the latter is written in the form of eq.(2.20). In the path-integral approach 
to quantization the $\xi, \bar{\xi}$ variables have zero anticommutators. 'To sec how equivalent results are nevertheless obtained in this approach we choose the gange $e=1, \psi=\bar{\psi}=0$ in (2.13) and perform the fermion palh-integral. 'The result is the following effective action for $x^{i}(t)$ :

$$
S_{e f f}=\int_{0}^{1} d t\left\{\dot{x} p-\frac{1}{2} p^{2}-\left(q-\frac{d}{2}\right) f-i d \ln \operatorname{det}\left[1+i\left(\partial_{t}\right)^{-1} f\right]+\text { const. }\right\} .
$$

A careful evaluation of the determinant was given in [11]; there is a possible anomaly contribution from the linear term in $f$; i.e. the "fadpole" in the diagrammatic expansion. A quick, but non rigorous, method of calculating this diagram is to replace the encrgy sum by an integral, at the same time introducing a regulator mass $\mu$ for the resulting infrared divergence. The result is then

$$
i d\left[\int_{0}^{l} f(t) d t\right] \int_{-\infty}^{+\infty} \frac{d E^{\prime}}{2 \pi(E+i \mu)}
$$

After ultraviolet regulation by a cutoff $\Lambda$ the energy integral becomes

$$
\int_{-\Lambda}^{\Lambda} \frac{d E}{2 \pi(E+i \mu)}=-\frac{i}{2}(2 k-1)+O\left(\frac{\mu}{\Lambda}\right)
$$

where the arbitrary integer $k$ arises from a choice of branch of the logarithm appcaring in the evaluation of the integral. Hence the term lincar in $f$ in the effective action is

$$
(-q+k d) \int_{0}^{1} f(l) d l
$$

Under an $S O(2)$ transformation

$$
f \rightarrow f+i g^{-1} \partial_{\imath} g \quad \xi \rightarrow g \xi
$$

If $g$ is connected to the identity, the (,$-S$ term is invariant provided that $g(0)=g(1)$. This periodicity implies that there are global gauge transformations for which $g=e^{2 \pi i m t}$, $m \in \mathbf{Z}$. For such transformations

$$
\int_{0}^{1} f(t) d t \rightarrow \int_{0}^{1} f(t) d t-2 \pi m
$$


and hence we obtain the quantization condition

$$
q \in \mathbf{Z}
$$

The case for which $q=\frac{d}{2}$ (which is consistent only for $d$ even) is special. Only in this case is the $S O(2)$ symmetry of (2.13) a genuine "internal" symmetry for which the Lagrangian, as against the action, is invariant. The $q=\frac{d}{2}$ casc is also special in another respect; when $q=\frac{d}{2}$ the action (2.13) has additional invariances. Firstly, the $S O(2)$ invariance is extended to $O(2)$; quantum mechanically this corresponds to the invariance of the equations for the $\frac{d}{2}$-index antisymmetric tensor under the discrete duality transformation $F \rightarrow \star F$ (where $\star$ is the Ifodge dual). Sccondly, for $q=\frac{d}{2}$ the action has a rigid conformal invariance. This is also true for $N>2, d$ even, for which the $\left(q-\frac{d}{2}\right)$ term is necessarily absent. In terms of the conformal Killing vectors $k^{\mu}$ of Minkowski spacetime, which satisfy $k_{(\mu, \nu)}=\frac{1}{d} \eta_{\mu \nu} k_{, \rho}^{\rho}$, the infinitesimal conformal t,ransformations can be written as

$$
\begin{aligned}
& \delta x^{\mu}=k^{\mu} \quad \delta p_{\mu}=-k_{, \mu}^{\nu} p_{\nu}-\frac{i}{2} \lambda_{i}^{\rho} \lambda_{i}^{\sigma} k_{\rho, \sigma \mu} \quad \delta \lambda_{i \mu}=k_{[\mu, \nu]} \lambda_{i}^{\nu} \\
& \delta e=\frac{2}{d} e k_{, \mu}^{\mu} \quad \delta \psi_{i}=\frac{1}{d}\left(\psi_{i} k^{\mu}{ }_{, \mu}-e \lambda_{i}^{\nu} k_{, \mu \nu}^{\mu}\right) \quad \delta f_{i j}=i \frac{2}{d} \psi_{[i} \lambda_{j]}^{\mu} k_{, \nu \mu} .
\end{aligned}
$$

In the $d=2$ case there is of course an infinite number of conformal Killing vectors.

Observe that it is precisely when $q=\frac{d}{2}$ that the cquations (2.23) are conformally invariant wave equations. This is an cxample of a property of a field theory that has a counterpart in a similar property of the underlying point particle action. When $q \neq \frac{d}{2}$ the invariance of the action requires that $k_{, t \nu}^{\mu}=0$, which is satisfied by the Killing vectors that generate the rigid Poincaré invariance of the particle action, and by the conformal Killing vector that generates dilatations.

The extension of (2.13) to include a background gravitational ficld was given in [4] (for $q=\frac{d}{2}$ ) where it was also shown that the background is necessarily flat for $N>2$. In terms of the variables of $(2.14)$, the action is

$$
S=\int d l\left\{\dot{x}^{\mu} p_{\mu}+i \bar{\xi}^{a} \dot{\xi}^{b} \eta_{a b}-i \psi \bar{\xi}^{\mu}\left(p_{\mu}-\frac{i}{2} \omega_{\mu b c}\left[\xi^{b}, \bar{\xi}^{c}\right\}\right)\right.
$$




$$
\begin{aligned}
& -i \vec{\psi} \xi^{\mu}\left(p_{\mu}-\frac{i}{2} \omega_{\mu b c}\left[\xi^{b}, \bar{\xi}^{c}\right]\right)+f\left(\frac{1}{2}\left[\xi^{a}, \bar{\xi}^{b}\right] \eta_{a b}-\left(q-\frac{d}{2}\right)\right) \\
& \left.-\frac{1}{2} e g^{\mu \nu}\left(p_{\mu}-\frac{i}{2} \omega_{\mu a b}\left[\xi^{a}, \bar{\xi}^{b}\right]\right)\left(p_{\nu}-\frac{i}{2} \omega_{\nu c d}\left[\xi^{c}, \bar{\xi}^{d}\right]\right)\right\}
\end{aligned}
$$

where $\lambda^{a}=\lambda^{\mu} e_{\mu}^{a}$ with $e_{\mu}^{a}$ the viclbein salisfying $e_{\mu}^{a} e_{\nu}^{b} \eta_{a b}=g_{\mu \nu}$.

We shall now investigate the possibility of an electromagnetic background. We have to add to (1.1) the $N$-extended supersymmetric generalization of the Lorentz coupling $\int d t \dot{x}^{\mu} A_{\mu}$. This is

$$
S_{L}=\int d t\left(\dot{x}^{\mu} A_{\mu}-\frac{i}{2} c \lambda_{i}^{\mu} \lambda_{i}^{\prime} F_{\mu \nu}\right)
$$

Under the supersymmetry transformations of (1.1), which are (in the absence of a gravitational background)

$$
\begin{array}{lcl}
\delta x^{\mu}=i \alpha_{i} \lambda_{i}^{\mu} & \delta \lambda_{i}^{\mu}=-\alpha \alpha_{i} p^{\mu} & \delta p_{\mu}=0 \\
\delta e=2 i \psi_{i} \alpha_{i} & \delta \psi_{i}=\dot{\alpha}_{i}-f_{i j} \alpha_{j} & \delta J_{i j}=0
\end{array}
$$

the action $S_{L}$ has the variation

$$
\delta S_{L}=i \alpha_{i} \lambda_{j}^{\mu}\left(\psi_{j} \lambda_{i}^{\nu}-\psi_{i} \lambda_{j}^{\nu}\right) F_{\mu \nu}-\frac{i}{2} c \alpha_{j} \lambda_{i}^{\mu} \lambda_{i}^{\nu} \lambda_{j}^{\rho} \partial_{\rho} F_{\mu \nu}
$$

For $N=1$ the first term vanishes identically and the second term by the Banchi identity $\partial_{[\rho} F_{\mu \nu]}=0$. For $N \geq 2$ the first term vanishes if and only if $F_{t t \prime}=0$. Thus an electromagnetic coupling is not possible for $N \geq 2$. This was to be expected from the well-known consistency problems with charged spin 1 fields.

Finally, we remark that the particle's wave function is, a priori, complex. 'l'he worldline time-reversal invariance of the action implics the existence of an antiunitary operator $K$ that leaves the constraints invariant. For the spin $\frac{1}{2}$ particle it was shown in [10] that

$$
K|\Psi\rangle=|\Psi\rangle
$$

implies that $\Psi$ is Majorana. On general grounds $K^{2}= \pm 1$ and (2.37) is consistent only when $K^{2}=+1$. As expected $K^{2}=-1$ whenever $\Psi$ cannot be Majorana. The doubling 
of $\Psi$ in this case can thercfore be thought of as an example of Kramer's degeneracy in quantum mechanics. For gencral $N$, we can write $K$ as

$$
K=\left(\otimes^{N} U\right) K_{0}
$$

where $K_{0}$ is the (basis - dependent) complex conjugation operator and $U$ is a unitary matrix acting on $H_{1}$ with the property that $U^{*} U= \pm 1$. Therefore for $N$ odd $K^{2}= \pm 1$ whereas for $N$ even $K^{2}=+1$, necessarily. For $N$ even it is therefore always possible to require of the wave function that it be real. In particular, this means that the antisymmetric tensors discussed in this paper can always be chosen to be real.

\section{3. $\quad N=2$ worldline supergravity in superspace}

In this section we present the superspace formulation of the $N=2$ model, thereby updating the results of [2] and generalizing the $N=1$ results of [10]. In flat superspace the covariant derivatives are

$$
D=i\left(\partial_{0}-\frac{i}{2} \vec{\theta} \partial_{t}\right) \quad \bar{D}=i\left(\partial_{\bar{\theta}}-\frac{i}{2} \theta \partial_{t}\right),
$$

which satisfy

$$
\{D, \ddot{D}\}=i \partial_{t} \quad D^{2}=D^{2}=0
$$

In a general superspace we denote a supervecter $V$ in a condinate basis by $V^{M}=$ $\left(v^{t}, v^{\theta}, v^{\bar{\theta}}\right)$ and in a tangent space basis by $V^{A}=\left(V^{+1}, V^{-1}, V^{-1}\right)$ with the summation convention

$$
V^{A} U_{A}=V^{0} U_{0}+V^{1} U_{1}-V^{i} U_{i}
$$

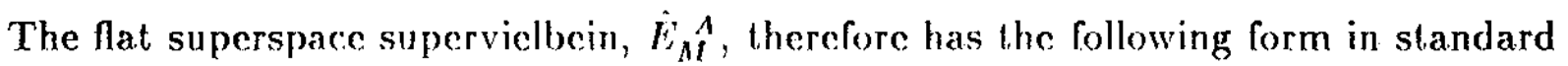
coordinates

$$
\hat{E}_{t}^{0}=1 \quad \hat{E}_{t}^{1}=0 \quad \hat{E}_{t}^{\top}{ }^{\bar{\top}}=0
$$




$$
\begin{array}{lll}
\hat{E}_{\theta}{ }^{0}=\frac{1}{2} \bar{\theta} & \hat{E}_{\theta}{ }^{1}=i & \dot{E}_{\theta}{ }^{\overline{1}}=0 \\
\hat{E}_{\bar{\theta}}{ }^{0}=\frac{1}{2} \theta & \hat{E}_{\bar{\theta}}{ }^{1}=0 & \dot{E}_{\hat{\theta}}{ }^{1}=-i
\end{array}
$$

We take the tangent space group to be $U(1)$, which acts by

$$
\delta V^{A}=V^{B} L_{B}^{A}
$$

with

$$
L_{1}^{1}=i l \quad L_{i}^{\top}=i l,
$$

and all others zero. With this structure it is easy to show that the imposition of standard conventional constraints implies that a general superspace of this lype is in fact fat;

$$
T_{11}^{0}=-i
$$

with all other components of $T_{A B} C^{\prime}$ vanishing. These constraints are invariant under the super Weyl transformations

$$
\begin{gathered}
E_{M}{ }^{0} \rightarrow S E_{M}{ }^{0} \\
E_{M}{ }^{1} \rightarrow S^{\frac{1}{2}}-i S^{-\frac{1}{2}} E_{M}{ }^{0} D_{\mathrm{i}} S,
\end{gathered}
$$

where $S$ is a real scalar superficld.

Since the superspace is flat we could choose a gauge in which the supervielbein takes the standard form (modulo moduli). In order to make contact with the component formalism we instead make the partial gauge choice for which $E_{M}^{A}$ has its superconformally flat form, i.e. $E_{M}^{A}$ differs from $\hat{E}_{M f}^{A}$ by a superconformal transformation with parameter $V$. We then find

$$
\begin{aligned}
& \left.D_{1}=V^{-\frac{1}{2}} D \quad D_{\overline{\mathrm{L}}}=V^{-\frac{1}{2}} \ddot{D}\right) \\
& D_{0}=V^{-1} \partial_{t}+\frac{i}{2} V^{-2}(\bar{D} V D+D V \bar{D})
\end{aligned}
$$


This gauge choice is preserved under a general superspace coordinate transformalion, with parameter $\xi^{A}$, provided that

$$
D_{A} \xi^{B}+\xi^{C} T_{C A}^{B}+L_{A}^{B}=I_{A}^{B}\left(S=V^{-1} \delta V\right)
$$

where $H_{A}{ }^{B}(S)$ is the infinitesimal form of the super Weyl transformation, i.e.

$$
\delta E_{M}^{A}=E_{M}^{B} I_{B}^{A}(S)
$$

as can be derived from (3.8). Onc finds

$$
\begin{aligned}
& \xi^{1}=-i D_{\overline{1}} \xi^{0} \quad V^{-1} \delta V=\frac{1}{2} D_{0} \xi^{0} \\
& L=\frac{i}{2}\left[D_{1}, D_{\overline{1}}\right] \xi^{0}
\end{aligned}
$$

which implies that

$$
\delta V=\frac{1}{2}\left[\partial_{t}+i V^{-2}(\bar{D} V D+D V \bar{D})\right] \xi^{0}
$$

The action for a set of $d$ scalar superficlds $\Phi^{\mu}$ coupled to supergravily is given by

$$
S=-2 \int d l d^{2} \theta E D_{1} \Phi^{\mu} D_{\mathfrak{i}} \omega^{\nu} \eta_{\mu \nu}
$$

which, in the superconformal gauge, is simply

$$
S=-2 \int d t d^{2} \theta V^{-1} D \Phi \bar{D} \Phi
$$

Integrating over $\theta$ and $\bar{\theta}$ and eliminating the auxiliary ficld, which is the $\theta \bar{\theta}$ component of $\Phi$, we obtain agreement with the component action upon making the identifications

$$
\begin{gathered}
e=V\left|\quad \Psi=-\frac{1}{\sqrt{2}} V \bar{D} V\right| \quad f=\frac{1}{2}[D, \bar{D}] \ln V \mid \\
x^{\mu}=\Phi^{\mu}\left|\quad \xi^{\mu}=\sqrt{2} V^{-\frac{1}{2}} D \Phi^{\mu}\right|
\end{gathered}
$$


where the vertical bar indicates that the superficld is to be evaluated at $\theta=\bar{\theta}=0$. From the equation for $f$ we sce that the superspace inlegrand of the Chern-Simons form is $-q \ln V$.

It is straightforward to couple the system to a mon-trivial gravitational field in this formalism; one simply replaces the flat Minkowski space metric $\eta_{\mu \nu}$ by a general metric $g_{\mu \nu}(\Phi)$ in $(3.14)$.

4. Massive antisymmetric tensor field equations from particle mechanics

If the background spacetime for the action (2.33) has isometries, generated by the Killing vectors $k_{(r)}^{\mu}$, then the action has corresponding rigid symmetries generated by the Noether charges

$$
G_{(r)}=k_{(r)}^{\prime \prime}(x) p_{n}
$$

These symmetries can be gauged by adding to the action the term

$$
S_{G}=\int d l l_{(r)} C_{(r)}
$$

Upon quantization the wave function must then satisfy the adelitional constraint

$$
k^{\prime \prime}(x) \partial_{\mu} \Psi(x)=0
$$

This is equivalent to a formulation of ficld theory in a lower dimensional spacetime. The simplest example occurs for a spacelime of the form (Ninkowski) ${ }_{d} \times S^{1}$, in which case $G=p_{d}$ is the charge associated with translations around the circle $S^{1}$. The constraint (4.3) becomes

$$
\partial_{m_{d}} \Psi(x)=0
$$

so that $\Psi$ depends only on $x^{\mu}, \mu=0, . ., d-1$, and the massless field equations in $(d+1)$ dimensions reduce to massless field equations of $d$-dimensional Minkowski space. This is 
just the usual dimensional reduction, but because $U(1)$ is $\Lambda$ belian it is possible to modify this procedure, as shown by Scherk and Schwarz [6]. In the context of particle mechanics the Scherk-Schwarz mechanism operates by the addition to the action of the term

$$
\int d t l(G+m)
$$

where $m$ is an arbitrary constant. Taking $G=p_{d}$ we find that the particle wave function must satisfy

$$
p_{d} \Psi=-m \Psi
$$

In this case the massless ficld equations in $(d+1)$ dimonsions (which are a consequence of the other constraints) will become massive ficld equations in $d$ dimensions*. Moreover, since the little group for massless particles in $(d+1)$ dimensions equals that for massive particles in $d$ dimensions, a pure spin $\frac{N}{2}$ massless ficld will always have just the right number of components for a pure spin $\frac{N}{2}$ massive ficld in one lower dimension.

Since the action (1.1) is applicable to massless ficlds with spin $\geq \frac{3}{2}$ only for $d$ cven, this mechanism allows us to oblain massive fields of arbitrary spin in odd dimensional spacetimes only. For $N=2$ there is no such restriction, however, and we can obtain massive antisymmetric tensor ficld equations for arbitrary $d$. The appropriate particle action is just (2.13) with the addition of the term (1.5) . Rather than add (4.5) we can simply solve the constraint by substituting $(-m)$ for $p_{d}$ wherever it appears in the rest of the action. In this way we arrive at the action

$$
\begin{aligned}
S= & \int d t\left\{\dot{x} \cdot p+i \bar{\xi} \cdot \dot{\xi}+i \dot{\zeta \zeta}-\frac{1}{2} e\left(p^{2}+m^{2}\right)-i \ddot{\psi}(\xi \cdot p-m \zeta)+\right. \\
& \left.-i \psi(\bar{\xi} \cdot p-m \bar{\zeta})+f\left(\frac{1}{2}[\xi, \bar{\xi}]+\frac{1}{2}[\zeta, \ddot{\zeta}]-q+\frac{d}{2}\right)\right\},
\end{aligned}
$$

* This mechanism has been used previously in the context of other particle models by Henneaux and Teitelboim [12] 
where $\zeta=\frac{1}{\sqrt{2}}\left(\lambda_{1}^{d}+i \lambda_{2}^{d}\right)$. The complex wave function satisfying the $S O(2)$ constraint can be written as

$$
\frac{1}{q !} \alpha^{\mu_{1}} \ldots \alpha^{\mu_{q}} F_{\mu_{1} \ldots \mu_{q}}+\frac{i m}{(q-1) !} \beta \alpha^{\mu_{1}} \ldots \alpha^{\mu_{q-1}} A_{\mu_{1} \ldots \mu_{q-1}}
$$

where $F$ and $A$ are, a priori, also complex, and $\beta$ is the anticommuting eigenvalue of $\zeta$, $\langle\beta| \zeta=\langle\beta| \beta$. The constraint $Q|\Psi\rangle=0$ imposed by $\bar{\psi}$ yiclds

$$
F_{\mu_{1} \ldots \mu_{q}}=q \partial_{\left[\mu_{1}\right.} A_{\left.\mu_{2} \ldots \mu_{q}\right]}
$$

which tells us that $A$ is the gauge potential for $F$. The constraint $\bar{Q}|\Psi\rangle=0$ then gives the antisymmetric tensor generalization of the Proca cyuation

$$
\partial^{\prime \prime} F_{\mu \nu_{1} \ldots \nu_{q-1}}-m^{2} A_{\nu_{1} \ldots \nu_{q-1}}=0
$$

The factor of $i$ in (4.8) is now seen to be necessary in order that $A$ may ullimately taken to be real. There is presumably some variant on the time reversal identification constraint $K|\Psi\rangle=|\Psi\rangle$ that could be imposed on the ware function $|\Psi\rangle$, ab initio, so as to ensure that $A$ is real, without having to impose it dircctly on 1 ifsell, but we have not investigated this point.

One question that may have occurred to the reader, concerning the general construction given above, is whether the addition of terms to the action of the form (4.2) could spoil symmetries that were present before their addition. It is readily checked for the example above that this does not happen. In the general case it is cnsured by the fact that the generators $G_{(r)}$ commute with the other gencrators. In the next section we will want to add generators that do not commute, but as long as we have a set of generators $\left\{\phi_{l}\right\}$ which close to form an algebra under the Poisson bracket,

$$
\left\{\phi_{l}, \phi_{m}\right\}_{P B}=f_{l m}^{n} \phi_{n},
$$


we can immediately write down a gauge invariant action*. To see this, consider a phase (super)space with coordinates $Y^{A}$ and let $\Omega$ be the supersymplectic form on phase space. Then the Poisson bracket is given by

$$
\{f, g\}_{P B}=-f \overleftarrow{\partial}_{A} \Omega^{A B} \vec{\partial}_{B} g
$$

with

$$
\begin{aligned}
& \Omega^{A B} \Omega_{B C}=\delta_{C}^{A} \\
& \Omega_{A B}=-(-1)^{a b} \Omega_{B A} .
\end{aligned}
$$

The action

$$
S=\int d t\left[-\frac{1}{2} \dot{Y}^{B} \Omega_{B A} Y^{A}-u^{\prime} \phi_{l}\right]
$$

has the gauge invariance

$$
\delta Y^{A}=-k^{l}\left(\phi_{1} \stackrel{\leftarrow}{\partial}_{B}\right) \Omega^{B A} \quad \delta u^{l}=-\dot{k}^{l}-u^{m} k^{n} f_{n m}^{l}
$$

It follows that the existence of a gauge invariant action is guarantecd once a set of constraints has been shown to form a closed algebra. What is not guarantecd is that the algebra of the quantum generators with respect to (anti)commutation will be the same as that of the classical generators with respect to the l'oisson brackets. We shall encounter examples where the classical and quantum algebras differ, but provided no new generators arise, and the algebra still closes, this causes no problems.

\section{Chirality and self-duality}

For d even a chirality constraint $\gamma_{\star} \Psi= \pm \Psi$ can be imposed on a Dirac spinor $\Psi$. Quantization of the $N=1$ particle model leads to a Dirac equation for a non-chiral

\footnotetext{
* This construction is well-known. See for example [13]
} 
spinor, but the model can be modified to incorporale a constraint which yields chiral wave-functions. Consider the classical quantity

$$
\Lambda_{1}=(2 i)^{\frac{d}{2}} \lambda^{0} \lambda^{1} \ldots \lambda^{d-1}
$$

Upon quantization we have

$$
\Lambda_{1} \rightarrow \hbar^{\frac{d}{2}} i^{\frac{d}{2}} \gamma^{0} \ldots \gamma^{d-1}=i h^{\frac{d}{2}} \gamma_{\star}
$$

To obtain a chirality constraint we should therefore add to the action a term of the form

$$
\int d t g_{1}(t)\left(\Lambda_{1}+i h^{\frac{d}{2}}\right)
$$

Variation of the Lagrange multiplier $g_{1}$ leads to the constraint $P_{+} \approx 0$, where

$$
P_{+}=\Lambda_{1}+i h^{\frac{d}{2}}
$$

This leads to the quantum mechanical constraint

$$
\frac{1}{2}\left(1+\gamma_{*}\right) \Psi=0
$$

The Lagrange multiplier term (5.3) is not real, and indecd $\left(1 \pm \gamma_{\star}\right)$ is not hermitian with respect to the Dirac: scalar product (although for Luclidcan signature this term would be real and the corresponding constraint hermitian). Howerer, this lack of reality is in a constraint term and does not present problems for the physical subspace.

The inclusion of the new constraint gencrales one further constraint, and the non-zero Poisson brackets of the complete set of constraint functions are

$$
\begin{gathered}
{[Q, Q]_{P B}=2 i I I \quad\left[P_{+}, Q\right]_{P B}=-2 i \tilde{Q} \quad[Q, \tilde{Q}]_{P B}=0} \\
{\left[P_{+}, \tilde{Q}\right]_{P B}=\left\{\begin{array}{ll}
-2 i Q, & \mathrm{~d}=2 \\
0, & d \geq 4
\end{array} \quad[\tilde{Q}, \tilde{Q}]_{P B}= \begin{cases}2 i I I, & \mathrm{~d}=2 \\
0, & d \geq 4\end{cases} \right.}
\end{gathered}
$$


where $\tilde{Q}$ is a new supersymmetry generator,

$$
\tilde{Q}=\frac{2 i^{\frac{d-2}{2}}}{(d-1) !} \varepsilon_{\mu_{1} \ldots \mu_{d}} \lambda^{\mu_{1}} \ldots \lambda^{\mu_{d-1}} p^{\mu_{d} t}
$$

and $Q$ and $I I$ are the original generators of supersymmetry and reparametrizations, respectively. Of course, we should now add to the action a Lagrange multiplier for $\tilde{Q}$.

Upon quantization

$$
\tilde{Q} \rightarrow \frac{1}{\sqrt{2}} \gamma_{\star} \gamma \cdot p
$$

and the quantum algebra is, for all even $d$,

$$
\begin{gathered}
{\left[P_{+}, Q\right]=\tilde{Q} \quad\left[I_{+}, \tilde{Q}\right]=Q \quad\{Q, \dot{Q}\}=0} \\
Q^{2}=H \quad \tilde{Q}^{2}=-I I
\end{gathered}
$$

For $d \geq 4$ there are therefore quantum corrections to the classical algebra. The quantum algebra is a non-compact $N=2$ extended supersymmetry algebra with an internal $O(1,1)$ symmetry group generated by $P_{+}$.

In the $N=2$ case, the analogue of the chirality constraint gives rise to (anti) self-dual antisymmetric gauge field strength tensors. The $N=2$ analugue of $\Lambda_{2}$ is, in terms of the complex $\boldsymbol{\xi}$ variables introduced in section 2 ,

$$
\Lambda_{2}=\frac{i^{n}}{(n !)^{2}} \varepsilon_{\mu_{1} \ldots \mu_{n} \nu_{1} \ldots \mu_{n}} \xi^{\prime \prime \prime} \ldots \xi^{\nu_{n}} \bar{\xi}^{n_{n}} \ldots \xi^{n_{1}}
$$

Quantum-mechanically, $\Lambda_{2}$ acting on an inhomogencous differential form annihilates all but the $n$-form contribution. On an $n$-form $F$ we have

$$
\Lambda_{2} F=i^{n} \star F
$$

where $\star$ is the Hodge dual operator

$$
(\star F)_{\mu_{1} \ldots \mu_{n}}=\frac{1}{n !} \varepsilon_{\nu_{1} \ldots \nu_{n} \mu_{1} \ldots \mu_{n}} F^{\nu_{1} \ldots \nu_{n}}
$$


Thus in order to impose the (anti)self-duality constraint,

$$
F= \pm i^{n-1} \star F
$$

we should add to the action the Lagrange mulliplicr term

$$
\int d i g_{2}(l)\left(\Lambda_{2} \mp i h^{\frac{d}{2}}\right)
$$

where $g_{2}$ is a new Lagrange multiplier. Clearly, it is only possible to impose reality and self-duality simultaneously in $d=2 \bmod 4$ (Minkowski spacc-time) dimensions. $\Lambda \mathrm{s}$ in the $N=1$ case the new constraint generates yet further constraints. The quantum algebra. of these constraints is most easily analysed in terms of differential forms. We have the correspondences

$$
\begin{array}{cc}
\xi^{\prime \prime} \leftrightarrow d x^{\mu} & \ddot{\xi}_{\mu} \leftrightarrow i_{\mu} \\
Q \leftrightarrow d \quad & \ddot{Q} \leftrightarrow d^{*} \\
I H \leftrightarrow d d^{*}+d^{*} d \equiv \Delta \quad M \leftrightarrow d x^{\mu} i_{\mu}-n,
\end{array}
$$

where $d$ is the exterior derivative operator, $i_{\mu}$ denotes the interior product to be taken with the basis vector $\frac{\partial}{\partial x^{\mu}}$ and $d x^{\mu} i_{\mu}$ on a $p-f o r m \omega_{p}$ gives $p \omega_{p}$. The operator $d^{*}$ is the adjoint of $d$ with respect to the scalar product

$$
\left(\Psi^{(1)}, \Psi^{(2)}\right)=\sum_{p=0}^{2 n} \int \bar{\Psi}_{p}^{(1)} \wedge \star \Psi_{p}^{(2)}
$$

where the wave functions are inhomogencous forms,

$$
\Psi^{(i)}=\sum_{p=0}^{2 n} \Psi_{p}^{(i)} \quad \Psi_{p}^{(i)} \text { a p form }
$$

For 2n-dimensional Minkowski space

$$
d^{*}=\star d \star
$$


We introduce the operators $\tau_{p}$ by

$$
\tau_{p}= \begin{cases}i^{p(p-1)+n-1} \star & \text { on } p \text { forms } \\ 0 & \text { on } q \text { forms } q \neq p\end{cases}
$$

which have the following properties

$$
\tau_{2 n-p} \cdot \tau_{p}=1 \quad \tau_{p} d=-d^{*} \tau_{p-1} \quad \tau_{p} d^{*}=-d \tau_{p+1}
$$

The self-duality constraint is incorporated by cxtending the $N=2$ algebra generated by $Q, \bar{Q}, I$ and $M$ to include the operator

$$
P=1+\tau_{n}
$$

The following additional operators are then generated

$$
\begin{aligned}
& S_{1}=\tau_{n} d-d \tau_{n} \quad \bar{S}_{1}=\tau_{n} d^{*}-d^{*} \tau_{n} \\
& S_{2}=I_{n} d+d I_{n} \quad \ddot{S}_{2}=I_{n} d^{*}+d^{*} I_{n} \\
& K=\Delta I_{n}+\left(d d^{*}\right) I_{n+1}+\left(d^{*} \cdot d\right) I_{n-1}
\end{aligned}
$$

where $I_{p}$ is the projector onto p-forms. The complete algebra therefore has the generators ( $\left.H, M, P, K ; Q, \vec{Q}, S_{1}, \bar{S}_{1}, S_{2}, \bar{S}_{2}\right)$. The nom-zero (anti)commutation relations are

$$
\begin{gathered}
\{Q, \bar{Q}\}=\Delta \quad[M I, Q]=Q \quad[M, \ddot{Q}]=-\vec{Q} \\
{[P, Q]=S_{1} \quad\left[P, S_{1}\right]=S_{2} \quad\left[P, S_{2}\right]=S_{1}} \\
{[P, \bar{Q}]=\bar{S}_{1} \quad\left[I, \bar{S}_{1}\right]=\bar{S}_{1}} \\
{\left[M, S_{1}\right]=S_{1} \quad\left[M, S_{2}\right]=S_{2}} \\
{\left[M I, \bar{S}_{1}\right]=-S_{1} \quad\left[M, \bar{S}_{2}\right]=-S_{2}} \\
\left\{S_{1}, \bar{S}_{1}\right\}=-K \quad\left\{S_{2}, \bar{S}_{2}\right\}=K
\end{gathered}
$$




$$
\left\{\bar{Q}, S_{2}\right\}=K \quad\left\{Q, \bar{S}_{2}\right\}=K
$$

Thus there are six (real) odd generators. 'The subalgebra generated by $S_{1}, S_{2}, M, P$ and $K$ is an $N=4$ supersymmetry algebra with an internal $O(2) \times O(1,1)$ symmetry.

In the $n=1$ case, $K$ equals $I I$ and $S_{2}$ coincides with $Q$. 'The algebra, which is then spanned by $Q, \bar{Q}, S_{1}, \bar{S}_{1}, M, P$ and $H$, is again an $N=1$ algebra with an $O(2) \times O(1,1)$ internal symmetry.

For $N \geq 2$ the analogue of $\Lambda_{1}$ and $\Lambda_{2}$ is

$$
\Lambda_{N} \propto \varepsilon_{\mu_{1} \nu_{1} \ldots \mu_{n} \nu_{n}}\left(\lambda_{i_{1}}^{\mu_{1}} \lambda_{i_{1}}^{\nu_{1}}\right) \ldots\left(\lambda_{i_{n}}^{\mu_{n}} \lambda_{i_{n}}^{\nu_{n}}\right)
$$

For $N$ even the imposition of the generalized self-duality constraint implies that the wave function (in tensorial form) is self-dual on cach block of $n$ indices; for $N$ odd the wave function is, in addition, constrained to be chiral. We have not attempted to work out the full algebra of constraints in this case.

\section{References}

[1] L. Brink, S. Deser, B. Zumino, P. di Vecthia and P.S. Howe, Phys. Lett. 64B (1976), 435; F.A. Berezin and N.S. Marinor, Ann. Phys. 104 (1977), 336

[ 2] L. Brink, P. di Vechia and P.S. Howe, Nucl. P'hys. B118 (1977), 76

[ 3] V.D. Gershun and V.I. Tkach JFTP Letl. 29 (1979), 320

[ 4] P.S. Ilowe, S. Penati, M. Pernici and P.K. 'Townsend, Phys. Lett. 215B (1988), 555

[5] R. Marnelius and U. Mârtensson, "BRSSl quartization of frec massless particles of arbitrary spin”, Univ. of Göleborg preprint 88-10.

[ 6] W. Siegel, Int. Journ. Mod. Phys. $\Lambda 3$ (1988) 2713; All free conformal representations in all dimensions, Stony Brook preprint ITP-SB-88-45 
[ 7] J. Scherk and J. Schwarz, Phys. Lell. 82B (1979), 60

[ 8] W. Siegel and B. Zwiebach, Phys. Lett. 184B (1987), 325

[ 9] A. Barducci, R. Casalbuoni, D. Dominici and L. Lusanna, Phys. Lett. 100B (1981), 126

[ 10] J.Henty, P.S. Howe and P.K. 'Townsend, Class. Quant. Grav. 5 (1988), 807

[11] S. Elitzur, E. Rabinovici, Y. Frishman and A. Schwimmer, Nucl. Phys. B273 (1986), 93

[12] M. Ilenneaux and C. Tcilclboim, First and second quantized point parlicles of any spin, to appear in the proccedings of the second mecting on Quantum Mechanics of Fundamental Systems, Dec. 1987, eds. C. Teitelboim and J. Zanelli, (Plenum Press) [ 13] W. Siegel, Phys. Lett. 203B (1988), 79 